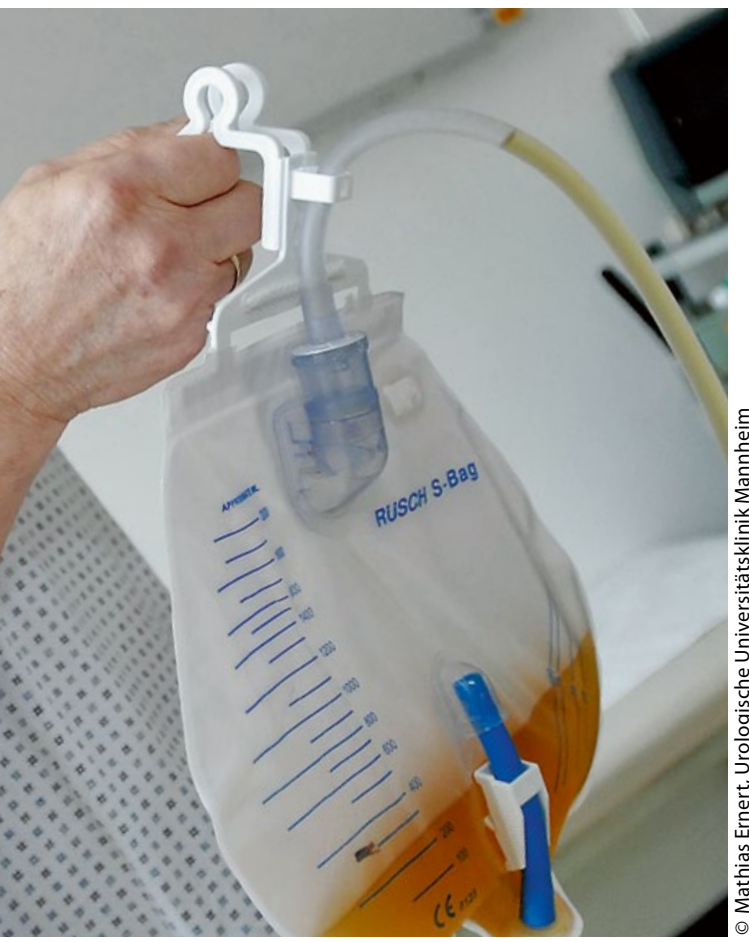

Vielfältige Infektionsmöglichkeiten ...
Urinkatheter

\section{Weniger Infektionen durch Prävention}

um $24 \%$ gesenkt werden - die anderen US-Kliniken erreichten nur $6 \%$.

Gravierendste Schwierigkeiten beim Umsetzen der Initiative waren mangelndes Engagement von Ärzten und Pflegepersonal, der Wunsch von Patient oder Familienmitgliedern nach einem Dauerkatheter sowie die häufige Katheterisierung der Patienten auf der Intensivstation. Mögliche Lösungswege sehen die Autoren in der Umsetzung eines geeigneten Urinmanagements, etwa durch geplante Toilettengänge, der ausführlichen Information von Patienten, Angehörigen und Klinikpersonal.

In Deutschland hat das Robert Koch-Institut Empfehlungen zur Prävention und Kontrolle katheterassoziierter Harnwegsinfektionen herausgegeben. Zudem existieren zum Thema Harnblasenkatheter die AWMFLeitlinien zur "Hygiene in Klinik und Praxis”.

Dr. Christine Starostzik

\section{latrogene Harnwegsinfekte}

\section{Antibiose vor Zystoskopie unnötig}

— Bei Patienten, die sich einer Zystoskopie unterziehen müssen und deren Urin vor der Untersuchung steril ist, senkt eine Antiobiotikaprophylaxe mit Levofloxacin im Vergleich zu Placebo nicht die Inzidenz von Harnwegsinfekten. Zu diesem Ergebnis kam eine kolumbianische Studie mit 285 ambulanten Patienten, die sich einer elektiven Zystoskopie unterziehen sollten. Alle Probanden hatten vor der Zystoskopie negative Urinkulturen. 30 bis 60 Minuten vor der Untersuchung erhielt die eine Hälfte der Studienteilnehmer 500 mg Levofloxacin oral, die andere Hälfte ein Placebo. Primärer Studienendpunkt war die Inzidenz von Harnwegsinfekten (HWI) drei bis zehn Tage nach der Zystoskopie, definiert als irritative Symptome vonseiten des Harntrakts zusammen mit einer positiven Urinkultur aus dem Mittelstrahlurin von $>10^{5}$ koloniebildenden Einheiten $(\mathrm{KbE}) / \mathrm{ml}$. Sekundärer Endpunkt war das Auftreten einer asymptomatischen Bakteriurie $>10^{5} \mathrm{KbE} / \mathrm{ml}$. Die HWI-Inzidenz betrug $0,7 \%$ in der Verum- und $3 \%$ in der Kontrollgruppe. Mit einem $p$-Wert von 0,17 hatte die Differenz keine statistische Aussagekraft. In puncto asymptomatischer Bakteriurie erreichte der Unterschied in der Inzidenz von 5,8\% (Verum) und 14,5\% (Placebo) $z w a r$ rechnerische Signifikanz $(p=0,01)$. Klinische Weiterungen zieht ein solcher Befund jedoch nicht nach sich, sofern der betreffende Patient keine weiteren endourologischen Prozeduren über sich ergehen lassen muss.

Dr. Robert Bublak

\section{Übergewicht und Krebs}

\section{BMl ohne Einfluss auf PCa-Aggressivität}

— Bei Männern, die an Prostatakrebs erkrankt und zudem übergewichtig sind, ist nicht davon auszugehen, dass ihre Erkrankung aggressiver verläuft. Eine intensivere Behandlung ist daher bei dicken Krebspatienten nicht erforderlich, wie die statistische Auswertung der Daten einer retrospektiven Studie vermuten lässt [Chamie K et al. Urology 2013; 81: 949-55].

In der statistischen Analyse wurden bei Prostatakarzinompatienten die PSA-Werte in einem Zeitraum von sieben Jahren vor der Biopsie ermittelt. Eine Stratifizierung nach vier BMI-Gruppen - also unter 25, zwischen 25 und 29,9, zwischen 30 und 34,9 sowie über 35 - ergab keinen signifikanten Unterschied zwischen den Gruppen beim Diabetesstatus, dem Präbiopsiewert des PSA, dem Gleason-Score sowie dem Tumorrisiko nach D'Amico. Wurden Alter, Ethnie und Gleason-Score herausgerechnet, gab es ebenfalls keinen signifikanten Zusammenhang zwischen BMI und dem PSA-Wert vor der Biopsie.

Die US-Wissenschaftler erinnern daran, dass bei dem bisher beobachteten Zusammenhang von Adipositas mit PSA und Schwere des Prostatakarzinoms oft der Zusammenhang mit kardiovaskulären Erkrankungen übersehen wird. So seien zum Beispiel in einer Studie mit Männern, die unterschiedlich aggressive Prostatakarzinome hatten, nur etwa $3 \%$ an den Folgen der Krebserkrankung gestorben, die meisten anderen Patienten an den Ursachen anderer Erkrankungen.

Peter Leiner 